\title{
Strong coupling as an interplay of quantum emitter hybridization with plasmonic dark and bright modes
}

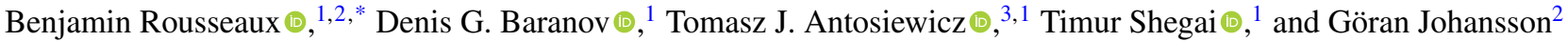 \\ ${ }^{1}$ Department of Physics, Chalmers University of Technology, 41296 Göteborg, Sweden \\ ${ }^{2}$ Department of Microtechnology and Nanoscience, MC2, Chalmers University of Technology, 41296 Göteborg, Sweden \\ ${ }^{3}$ Faculty of Physics, University of Warsaw, Pasteura 5, 02-093 Warsaw, Poland
}

(Received 24 October 2019; revised 10 April 2020; accepted 16 June 2020; published 13 July 2020)

\begin{abstract}
Strong coupling between a single quantum emitter and an electromagnetic mode is one of the key effects in quantum optics. In the cavity QED approach to plasmonics, strongly coupled systems are usually understood as single-transition emitters resonantly coupled to a single radiative plasmonic mode. However, plasmonic cavities also support nonradiative (or "dark") modes, which offer much higher coupling strengths. On the other hand, realistic quantum emitters often support multiple electronic transitions of various symmetries, which could overlap with higher order plasmonic transitions-in the blue or ultraviolet part of the spectrum. Here, we show that despite very large detuning between a bright mode and an excitonic transition, their strong coupling can be ensured by leveraging higher energy dark modes of the optical cavity. Specifically, when a dark mode interacts strongly with an excitonic transition, the lower polariton of the hybridized spectrum can be pushed to energies of the bright mode. The resulting interaction of the lower dark-mode-exciton polariton and bright mode yields significant vacuum Rabi splitting, which hinges on the existence of the dark mode. We develop a simple model illustrating the modification of the system response in the "dark" strong coupling regime and demonstrate single photon nonlinearity. These results may find important implications in the emerging field of room-temperature quantum plasmonics.
\end{abstract}

DOI: 10.1103/PhysRevResearch.2.033056

\section{INTRODUCTION}

Interaction of a quantum emitter (QE) with an optical cavity is at the heart of modern quantum optics. In the regime of weak QE-cavity coupling, the presence of a QE may be treated as a perturbation that affects the eigenmode of the cavity $[1,2]$. However, when the interaction between the cavity mode and the QE is strong enough, they form dressed polaritonic states (also called plexcitons [3]) separated by the vacuum Rabi splitting in the energy spectrum [4-7]. As the QE and the optical mode can no longer be treated as separate entities in this regime, such an evolution of the system not only modifies its optical response, but also dramatically affects exciton transport [8] and photochemical [9-13] properties.

Strong light-matter coupling is particularly interesting in the single-emitter limit, when unique features of the JaynesCummings ladder enable single-photon optical nonlinearities $[14,15]$. Rabi splitting between single quantum dots and dielectric high- $Q$ microcavities was observed in a number of works but only at cryogenic temperatures [16,17]. Plasmonic nanocavities enable observation of strong coupling with quantum dots and organic chromophores at room temperatures

\footnotetext{
*benjaminrousseaux@gmail.com
}

Published by the American Physical Society under the terms of the Creative Commons Attribution 4.0 International license. Further distribution of this work must maintain attribution to the author(s) and the published article's title, journal citation, and DOI. Funded by Bibsam.
[18-22], but most of such structures are at the border between the weak and the strong coupling regimes due to limited coupling strength [23].

The value of the coupling strength is determined by the transition dipole moment of the $\mathrm{QE}$ and the vacuum electric field of the cavity [4,6,7]. To achieve Rabi splitting in the visible range, the electronic transition of the $\mathrm{QE}$ has to be resonant with the bright mode of the cavity in the visible range. However, many material systems that are used to emulate QEs, for example, colloidal quantum dots [24,25] and excitons in transition-metal dichalcogenides monolayers [26], also possess electronic transitions at higher energies, which are often characterized by higher values of the oscillator strength. The high oscillator strength of these transitions could potentially be used to enhance the magnitude of Rabi splitting if the dipolar plasmon resonance can be tuned to the appropriate frequency range to overlap with those transitions. However, such an approach would require tuning dipolar plasmon resonances to the ultraviolet (UV) range, which has a number of disadvantages, including the complexity of optical measurements in this spectral range and the necessity of utilizing metals with significantly high plasma frequency, such as aluminium [27-29].

Alternatively, strong coupling between the QE and the so-called "dark," weakly radiative modes of conventional Ag and Au nanoparticles has been explored [30-37]. Despite the fact these dark modes are not observable using traditional optical techniques (although they can be observed by electron energy loss spectroscopy (EELS) [38-42], or in scattered light by large clusters [43] and anapoles [44]), it might be 
possible to visualize them by further hybridization of the dark mode-QE state with the bright mode of the resonator. We emphasize that the terminology of plasmonic "dark" modes has also been used for near-field plasmon modes of interacting nanoparticles, yielding zero net dipole moment [45]. In the weak coupling scenario, the interaction of a $\mathrm{QE}$ with a dark mode leads to quenching of emission [46,47], which is why these modes are often assumed to be detrimental for the purposes of vacuum Rabi splitting. In the strong coupling regime, however, Rabi splitting is relatively robust with respect to quenching when the emitter is spectrally tuned to the bright dipole mode of a plasmonic nanoparticle, as was shown recently [31]. It has also been shown that light-forbidden quadrupolar transitions of excitons coupled to a nanoparticle on mirror system can lead to strong coupling $[37,48]$.

In this work, we demonstrate theoretically that by coupling a high-energy transition of a QE to a cavity dark mode, it is possible to achieve observable Rabi splitting between two bright polariton modes. The dark mode plays a role of a tuning mechanism of the high-energy QE resonance toward the bright plasmon mode, where the interaction can take place. We analyze the system response with the use of a master equation approach and apply it to the case of a single QE coupled to the plasmonic modes of a metallic nanosphere. Note that all the parameter values correspond to a realistic geometry, thereby suggesting a practical recipe for the realization of vacuum Rabi splitting with a single $\mathrm{QE}$ at room temperature. Our results could potentially help in understanding the microscopic behavior of experimental observations of QE-plasmon systems such as those shown in Refs. [19,20], where a single emitter strong coupling was demonstrated but modeling the system with a single mode for the plasmonic response yields unrealistic values for the dipole moments of the QE [23].

\section{SINGLE EMITTER COUPLED TO A DARK AND A BRIGHT MODE}

The system under study is schematically shown in Fig. 1(a). It is composed of two cavity modes and a QE. One of the modes is denoted as bright ("B") since it has a low nonradiative loss rate $\gamma_{B}^{\text {nonrad }}$, while the other one is dark ("D") and has low radiative loss $\gamma_{D}^{\text {rad }}$, such that $\gamma_{D}^{\mathrm{rad}} / \gamma_{B}^{\mathrm{rad}} \ll 1$, and high nonradiative losses $\gamma_{D}^{\text {nonrad }}$. The choice of such a system is motivated by the existence of localized surface plasmon resonances of a metallic nanosphere: The lowest order mode has a dipolar nature and couples efficiently to light, while the higher order modes are strongly confined and appear only in the near field. The emitter couples to the bright mode and to the dark mode with coupling strengths $g_{B}$ and $g_{D}$, respectively. The energy diagram sketched in Fig. 1(b) elucidates the resulting interaction picture in this kind of system. The emitter interacts with the dark mode, resulting in two polariton modes separated by a "dark" Rabi splitting, which cannot be observed in the far field. The lower of these two polaritons, in turn, interacts with the bright cavity mode, leading to formation of another pair of polaritonic states, which can be observed in scattering owing to the radiative character of the bright mode.

We apply a simple analytical model based on a master equation with a weak drive to our system. This approach is



(b)

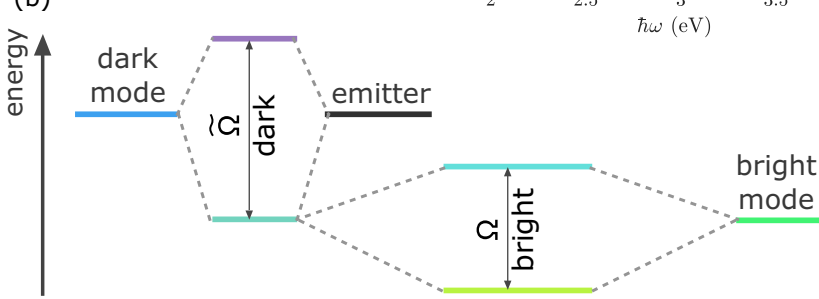

FIG. 1. (a) Schematic illustration of the system under study: a cavity with a bright mode and a dark mode couples to a $\mathrm{QE}$. (b) Sketch of the energy diagram of the three-component system. The dark mode-emitter coupling results in "dark" polaritons; the lower of those two in turn couples with the bright mode, resulting in two "bright" polaritons that can be resolved in the scattering spectrum. (c) Spontaneous emission enhancement factor (or Purcell factor) for a 10-nm $\mathrm{Ag}$ nanosphere, $1 \mathrm{~nm}$ away from the surface (see Appendix B for details). The green line shows the dipole mode contribution, while the dashed line is the dark pseudomode contribution.

also fully equivalent to the temporal coupled mode theory in evaluating the scattering dynamics $[49,50]$ (see Appendix A).

\section{A. Master equation in the weak pumping limit}

We use a master equation approach to calculate the scattering spectra in the main text. This approach not only corresponds to classical spectra in the weak pumping limit but allows the modeling of quantum nonlinearities such as saturation of the bright mode that arise in the strong pumping limit. Here, we limit our study to the weak pumping limit and show the photon blockade by calculating the photon statistics of the scattered signal. The Hamiltonian of the QE-two mode cavity system in the rotating-wave approximation is

$$
\begin{aligned}
\widehat{H}_{S}= & \omega_{E} \widehat{\sigma}_{+} \widehat{\sigma}_{-}+\omega_{B} \widehat{a}_{B}^{\dagger} \widehat{a}_{B}+\omega_{D} \widehat{a}_{D}^{\dagger} \widehat{a}_{D} \\
& +g_{B}\left(\widehat{a}_{B}^{\dagger} \widehat{\sigma}_{-}+\widehat{a}_{B} \widehat{\sigma}_{+}\right)+g_{D}\left(\widehat{a}_{D}^{\dagger} \widehat{\sigma}_{-}+\widehat{a}_{D} \widehat{\sigma}_{+}\right),
\end{aligned}
$$

corresponding conceptually to the system described in Fig. 2. $\omega_{E, B, D}$ here denote the transition frequencies of the $\mathrm{QE}$, the bright mode, and the dark mode, respectively. $\widehat{a}_{B, D}$ are the anniliation operators for the bright and dark modes, respectively, and $g_{B, D}$ are their corresponding coupling strengths with the QE. The master equation with a drive term is

$$
\begin{gathered}
\dot{\varrho}=-i[\widehat{H}, \widehat{\varrho}]+\sum_{j=B, D} \gamma_{j}\left(\widehat{a}_{j} \widehat{\varrho} \widehat{a}_{j}^{\dagger}-\frac{1}{2} \widehat{\varrho} \widehat{a}_{j}^{\dagger} \widehat{a}_{j}-\frac{1}{2} \widehat{a}_{j}^{\dagger} \widehat{a}_{j} \widehat{\varrho}\right), \\
\widehat{H}=\widehat{H}_{S}+\widehat{H}_{\text {drive }} \\
\widehat{H}_{\text {drive }}=-\left[\frac{\mu_{E}}{\hbar}\left(\widehat{\sigma}_{-}+\widehat{\sigma}_{+}\right)+\frac{\mu_{B}}{\hbar}\left(\widehat{a}_{B}+\widehat{a}_{B}^{\dagger}\right)\right] E_{L} \cos \omega t,
\end{gathered}
$$




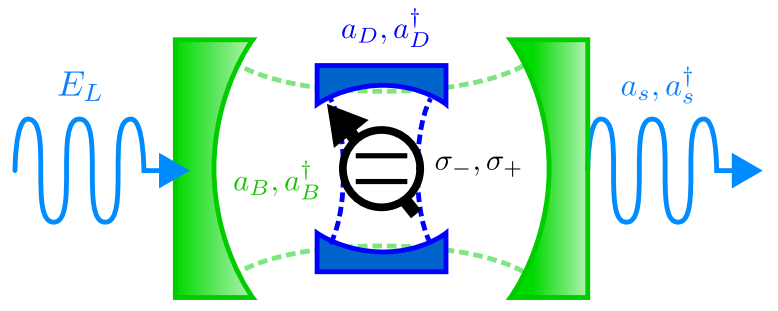

FIG. 2. Sketch of the model corresponding to Hamiltonian (1). A single two-level QE with lowering and raising operators $\widehat{\sigma}_{-}, \widehat{\sigma}_{+}$ is coupled to a dark cavity mode with annihilation and creation operators $\widehat{a}_{D}, \widehat{a}_{D}^{\dagger}$ and to a bright cavity mode with annihilation and creation operators $\widehat{a}_{B}, \widehat{a}_{B}^{\dagger}$. The system is driven with a laser field $E_{L}$ and the output field $\widehat{a}_{s}$ from (8) contains components from both the $\mathrm{QE}$ and the bright mode.

where $\widehat{\varrho}$ is the density operator for the emitter-bright-modedark-mode system, $\gamma_{j}=\gamma_{j}^{\text {rad }}+\gamma_{j}^{\text {non-rad }}, j=B, D$ are the loss rates of the bright and dark modes, respectively, $\mu_{E, B}$ are the dipole moments of the emitter and the bright mode, respectively (we neglected the pumping term of the dark mode since it couples only locally to the $\mathrm{QE}$ ), and the system is driven with a laser field amplitude $E_{L}$ and frequency $\omega$. Writing the Hamiltonian in the rotating frame of the driving field and applying the rotating-wave approximation yield the Hamiltonian in the form

$$
\begin{aligned}
\widehat{H}= & \Delta_{E} \widehat{\sigma}_{+} \widehat{\sigma}_{-}+\Delta_{B} \widehat{a}_{B}^{\dagger} \widehat{a}_{B}+\Delta_{D} \widehat{a}_{D}^{\dagger} \widehat{a}_{D} \\
& +g_{B}\left(\widehat{a}_{B}^{\dagger} \widehat{\sigma}_{-}+\widehat{a}_{B} \widehat{\sigma}_{+}\right)+g_{D}\left(\widehat{a}_{D}^{\dagger} \widehat{\sigma}_{-}+\widehat{a}_{D} \widehat{\sigma}_{+}\right) \\
& +\frac{\mathcal{E}_{E}}{2}\left(\widehat{\sigma}_{-}+\widehat{\sigma}_{+}\right)+\frac{\mathcal{E}_{B}}{2}\left(\widehat{a}_{B}+\widehat{a}_{B}^{\dagger}\right),
\end{aligned}
$$

where $\Delta_{j}=\omega_{j}-\omega$ and $\mathcal{E}_{j}=-\mu_{j} E_{L} / \hbar, j=E, B, D$. Since we study the weak pumping regime, the system is rarely in an excited state and thus the $\widehat{a}_{j} \widehat{\varrho} \widehat{a}_{j}^{\dagger}$ terms in the master equation can be neglected. This is equivalent to considering the effective Schrödinger equation

$$
i \frac{d|\psi\rangle}{d t}=\widetilde{H}|\psi\rangle,
$$

with $\widetilde{H}=\widehat{H}-i \frac{\gamma_{E}}{2} \widehat{\sigma}_{+} \widehat{\sigma}_{-}-i \frac{\gamma_{B}}{2} \widehat{a}_{B}^{\dagger} \widehat{a}_{B}-i \frac{\gamma_{D}}{2} \widehat{a}_{D}^{\dagger} \widehat{a}_{D}$ and whose steady-state solution yields the scattering spectrum and the photon statistics for zero delay. To solve this equation in the weak pumping limit, we proceed as in Refs. $[48,51]$ and solve for the steady state:

$$
\begin{aligned}
\left|\psi_{\text {s.s. }}\right\rangle & =\sum_{a=g, e} \sum_{b, c=0}^{2} c_{a, b, c}|a, b, c\rangle \\
\widetilde{H}\left|\psi_{\text {s.s. }}\right\rangle & =0,
\end{aligned}
$$

where we truncate the bright and dark excitation basis to 2 , which is needed to evaluate the second-order correlation function.

\section{B. Scattering spectrum and photon statistics}

The scattering spectrum is obtained by constructing the scattering operator,

$$
\widehat{a}_{s}=\mu_{E} \widehat{\sigma}_{-}+\mu_{B} \widehat{a}_{B},
$$

and computing the average over the steady-state of the associated number operator,

$$
S(\omega)=\left\langle\psi_{\text {s.s. }}\left|\widehat{a}_{s}^{\dagger} \widehat{a}_{s}\right| \psi_{\text {s.s. }}\right\rangle .
$$

When we consider the scattering map versus the nanosphere radius $R$, one should include the radius dependence of the scattered operator since larger nanospheres have faster radiative decay rates. To account for the radius dependence, we use the radiative decay rate formula from Ref. [52]:

$$
\gamma_{B}^{\mathrm{rad}}=4 \varepsilon_{b}^{3 / 2}\left(\frac{\omega_{B} R}{c}\right)^{3}\left[\frac{\partial}{\partial \omega} \operatorname{Re}\left\{\varepsilon_{m}(\omega)\right\}\right]_{\omega=\omega_{B}}^{-1},
$$

with $\varepsilon_{b}$ being the dielectric function of the surrounding medium, $\omega_{B}$ being the surface plasmon resonance frequency of the nanoparticle (here considering $\mathrm{Ag}$ ), $R$ being the radius of the nanoparticle, and $\varepsilon_{m}(\omega)$ being its Drude permittivity. The transition dipole moment $\mu_{B}$ is then given as a function of the radiative decay rate through the Fermi golden rule formula:

$$
\mu_{B}=\sqrt{\frac{3 \hbar \pi \epsilon_{b} c^{3}}{\omega_{B}^{3}} \gamma_{B}^{\mathrm{rad}}} .
$$

The photon statistics of the scattered signal is studied through the second-order correlation function for zero time delay [51]:

$$
g_{\omega}^{(2)}(0)=\frac{\left\langle\psi_{\text {s.s. }}\left|\widehat{a}_{s}^{\dagger} \widehat{a}_{s}^{\dagger} \widehat{a}_{s} \widehat{a}_{s}\right| \psi_{\text {s.s. }}\right\rangle}{\left\langle\psi_{\text {s.s. }}\left|\widehat{a}_{s}^{\dagger} \widehat{a}_{s}\right| \psi_{\text {s.s. }}\right\rangle^{2}} .
$$

The numerator of this function describes the probability of detecting two simultaneous scattered photons in a HanburyBrown-Twiss setup. The scattering of a laser beam on a classical system yields the coherent state value of 1 for $g_{\omega}^{(2)}(0)$, while antibunched light corresponding to the quantized nature of the scattered radiation yields values below 1 , and bunched light such as thermal sources or photon pair sources yields values above 1 .

\section{Bright mode-dark plexciton vacuum Rabi splitting}

We begin our study with a quick calculation of the scattering, considering a cavity with the bright mode at $3 \mathrm{eV}$ and the dark pseudomode at $3.4 \mathrm{eV}$, corresponding to an $\mathrm{Ag}$ nanosphere of $10 \mathrm{~nm}$ diameter [see Fig. 1(c) and Appendix B for the Purcell factor calculation]. We used a local-response approximation for a silver nanosphere for simplicity, but this scheme could apply to nonlocal-corrected models where the dark pseudomode is replaced by the quadrupolar mode [53]. Also, nonlocal effects were recently shown to have negligible effect on vacuum Rabi splittings [41,54], and modeling of strongly coupled Al-benzene systems showed an equally strong hybrid modes [29]. For the bright- and dark-mode linewidths, we use $\gamma_{B}^{\text {rad }}=\gamma_{D}^{\text {non-rad }}=0.1 \mathrm{eV}$. Furthermore, we here assume $\gamma_{D}^{\text {rad }}=\gamma_{B}^{\text {nonrad }}=0$. We emphasize that in this first calculation, we fixed these decay rate values arbitrarily. Later, we compute these values for the case of an $\mathrm{Ag}$ nanosphere whose dielectric constant is taken from Ref. [55]. To strengthen our motivation, we examine which QEs might be suitable for the proposed strong coupling scheme. Colloidal quantum dots (QDs), such as CdSe QDs, have a transition dipole moment of about 5-15 D at the wavelength of 
(a)

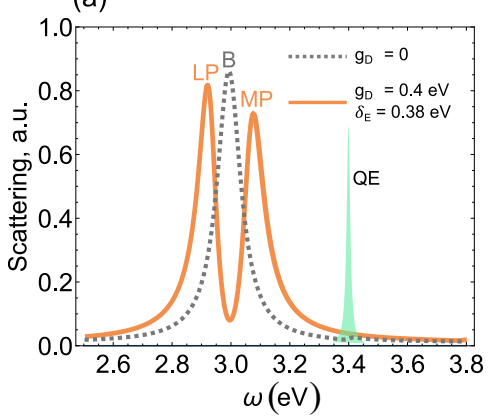

(b)

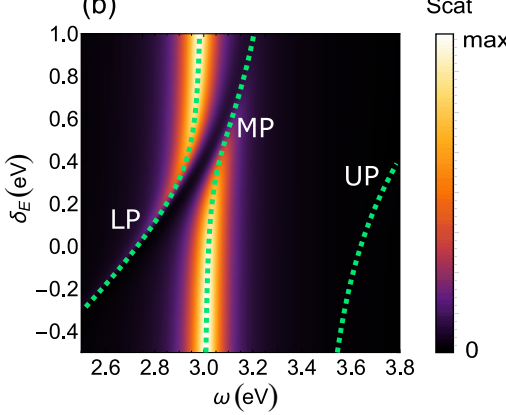

(c)

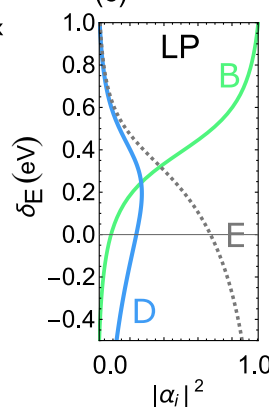

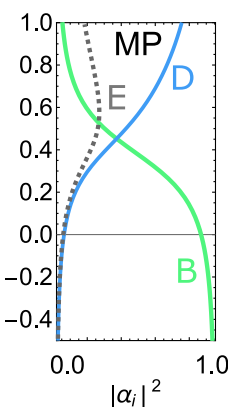

FIG. 3. Theoretical modeling of a generic coupled three-component system. (a) Scattering spectrum by the $3 \times 3$ system with the exemplary parameters outlined in the text in the absence of the dark mode, $g_{D}=0$ (dashed), and in the presence of the dark-mode-emitter coupling, $g_{D}=0.4 \mathrm{eV}$ (solid), and the bright-mode-emitter detuning, $\delta_{E}=0.38 \mathrm{eV}$. The filled curve depicts spectral position of the QE transition at $3.4 \mathrm{eV}$. (b) Calculated scattering spectra of the exemplary $3 \times 3$ system as a function of the emitter detuning $\delta_{E}$ for fixed $g D=0.4 \mathrm{eV}$. Dashed lines depict the real parts of the lower, middle, and upper polariton frequencies of the $3 \times 3$ system. (c) Absolute squared values of Hopfield coefficients $\left|\alpha_{B}\right|^{2},\left|\alpha_{D}\right|^{2}$, and $\left|\alpha_{E}\right|^{2}$ of the lower (left) and middle (right) polaritons vs emitter detuning $\delta_{E}$ for $g_{D}=0.4 \mathrm{eV}$.

$600 \mathrm{~nm}$ [56]. At the same time, these QDs are known to have high absorption and extinction coefficients in the UV range, exceeding that in the visible range by at least an order of magnitude [24,25]. Recalling that the extinction cross section of a two-level system under weak excitation is related to its transition dipole moment $\mu$ via $\sigma_{\text {ext }}=\omega_{E} \mu^{2} /\left(\hbar c \varepsilon_{0} \gamma_{E}\right)$ [24], where $c$ is the speed of light and $\varepsilon_{0}$ is the vacuum permittivity, and by assuming that the absorption peak predominantly originates from a single electronic transition (which might be not true in a realistic system), we may estimate the dipole moment of the UV transition is of the order of $100 \mathrm{D}$. Based on this simple estimation, we assign $g_{B}=0.1 \mathrm{eV}$ and $g_{D}=$ $0.4 \mathrm{eV}$, corresponding to a point emitter located $1 \mathrm{~nm}$ from the surface of the Ag nanosphere. According to Fermi's golden rule formula for the radiative decay rate, this value of the transition dipole moment results in $\gamma_{E}^{\mathrm{rad}} \approx 3 \mu \mathrm{eV}$, which is negligible in comparison to other decay rates.

To gain initial understanding of the three-component system behavior, we examine in Fig. 3(a) how the presence of the dark mode affects the elastic scattering spectrum for the QE tuned to the dark mode energy of $3.4 \mathrm{eV}$ in accordance with Eqs. (1) and (2). When the dark mode is turned off, $g_{D}=0$, the scattering spectrum exhibits one prominent peak corresponding to the uncoupled bright mode. However, when the coupling to the dark mode is introduced via $g_{D}$, the scattering spectrum presents two peaks around $3 \mathrm{eV}$, suggesting the onset of strong coupling between the emitter and the bright mode.

In order to corroborate the strong coupling regime upon coupling to the dark mode, we analyze the elastic scattering from the system versus the QE detuning $\delta_{E}=\omega_{E}-\omega_{B}$, Figs. 3(b). As one can see, an anticrossing occurs when the $\mathrm{QE}$ frequency crosses the dark mode frequency $\left(\delta_{E} \approx 0.4 \mathrm{eV}\right)$, i.e., at $\omega_{E} \approx \omega_{D}$. Notably, the Rabi splitting itself still occurs at the frequency of the unperturbed bright mode around $3 \mathrm{eV}$. The scattering peaks precisely follow eigenenergies of the Hamiltonian [Eq. (A2)], which are shown by the dashed lines in Fig. 3(b). The anticrossing of the eigenvalues confirms the strong coupling regime in the system. This is the main result of our paper that we would like to emphasize: One can leverage high transition dipole moments of certain QEs typically lying in the UV region to observe Rabi splittings in the visible range, provided that the emitter additionally interacts with a high-energy nonradiative mode.

Eigenvectors of Hamiltonian (Eq. (A2)) correspond to three-component quasiparticles: $\left|E P_{i}\right\rangle=c_{B}|B\rangle+c_{D}|D\rangle+$ $c_{E}|E\rangle$, where $|B\rangle,|D\rangle$, and $|E\rangle$ denote the bare bright mode, dark mode, and QE states, respectively. The lowest, medium, and highest energy solutions are referred to as the lower, middle, and upper polaritons (LP, MP, UP), respectively. Absolute amplitudes of these contributions (Hopfield coefficients), shown in Fig. 3(c) for the LP and MP as a function of the $\mathrm{QE}$ detuning, confirm that both bright polaritons have contributions from the bright and dark modes as well as the QE at the avoided crossing position and thus indeed present mixed light-matter states. The "bright" Rabi splitting observed in the spectra around $3 \mathrm{eV}$ occurs between the LP and MP. Neglecting losses, we can obtain an analytical expression for the magnitude of this splitting from the $3 \times 3$ Hamiltonian (see Appendix $\mathrm{C}$ for the derivation of this formula):

$$
\Omega_{\text {bright }}=\sqrt{2} g_{B} \sqrt{1+\frac{\omega_{D}-\omega_{E}}{\sqrt{4 g_{D}^{2}+\left(\omega_{D}-\omega_{E}\right)^{2}}}} .
$$

As one can see, it is mostly affected by the bright-emitter coupling constant $g_{B}$, which is determined by the transition dipole moment of the emitter and the vacuum electric field of the lower energy bright mode [4,7]. The dark-emitter coupling constant $g_{D}$, at the same time, has a negative effect on the resulting splitting. However, it is the large coupling to the dark mode that allows to effectively "push" the QE resonance down to the visible region, where it can interact with the bright mode. Looking at expression (13), one should also note that even if $g_{D}$ reduces the Rabi splitting, it can only bring it down to $\sqrt{2} g_{B}$ when $g_{D} \rightarrow \infty$. This role of the dark mode-emitter coupling can be illustrated by the expression for the optimal emitter-bright-mode detuning $\delta_{E}^{\text {opt }}$, upon which the bright mode is in zero detuning with the polariton formed by the dark mode-QE coupling (see Appendix C):

$$
\delta_{E}^{\mathrm{opt}}=g_{D}^{2} /\left(\omega_{D}-\omega_{B}\right) .
$$



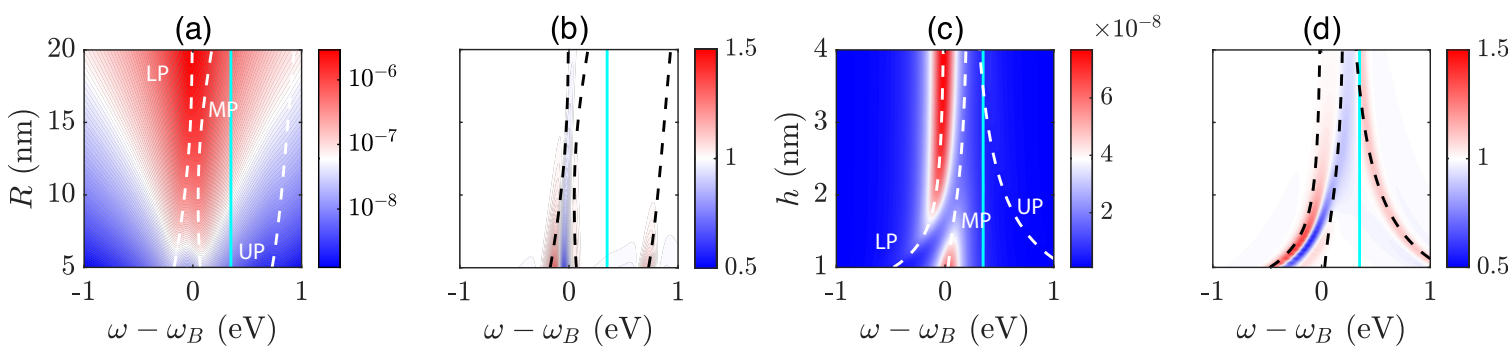

FIG. 4. Strong dark coupling in an Ag nanosphere. (a) False-color map of calculated scattering spectra of a $100 \mathrm{D}$ point dipole QE 1.5 $\mathrm{nm}$ away from the surface of an Ag nanosphere of radius $R$. The emitter frequency is set to $\omega_{E}=\omega_{D}, 0.35 \mathrm{eV}$ away from the bright mode (cyan vertical line). For better visibility, this map is shown in log scale. (b) Second-order correlation function $g_{\omega}^{(2)}(0)$ vs $R$ for $h=1.5 \mathrm{~nm}$. (c) Calculated scattering spectra for the same system vs the emitter-surface distance $h$ for $R=5 \mathrm{~nm}$. (d) Second-order correlation function $g_{\omega}^{(2)}(0)$ vs $h$ for $R=5 \mathrm{~nm}$.

Essentially, this equation shows that the larger the dark modeemitter coupling is, the higher $\omega_{E}$ should be in order for its hybridized resonance to overlap perfectly with the bright mode in the visible region. To underline the importance of the strong QE-dark mode interaction, we also provide the scattering map when $g_{B}=0$ as well as the absorption map for the same parameters as in Fig. 3(b), in Appendix C.

\section{Single $Q E$ in the vicinity of a silver nanosphere}

We further elaborate the concept of dark strong coupling by inspecting the response of a specific nanocavity, with the use of the master equation approach derived in the previous paragraphs. We choose a silver spherical nanoparticle of radius $R$ and a QE placed at a distance $h$ from the nanosphere surface. As was mentioned above, the dipole moment of the UV transition of some QEs could reach $100 \mathrm{D}(\approx 2 \mathrm{e} \mathrm{nm})$, and the total decay rate of such a transition could be of the order of $0.1 \mathrm{eV}$. We also compute all relevant parameters $\omega_{j}, \gamma_{j}, g_{j}, j=B, D$ from a Lorentzian fit of a Green's tensor approach [57], while $\gamma_{B}^{\mathrm{rad}}$ is taken from (10) and $\gamma_{D}^{\mathrm{rad}}$ is still assumed to be zero. The dielectric function of $\mathrm{Ag}$ is taken from Ref. [55], accounting for realistic values of the nonradiative decay. For radii $R$ between 5 and $20 \mathrm{~nm}$, we find $\gamma_{B}^{\text {rad }}$ between 0.1 and $5 \mathrm{meV}$, while the full decay rates $\gamma_{B}, \gamma_{D} \approx 0.3 \mathrm{eV}$, which is typical of small nanospheres whose field response is dominated by absorption. The map of elastic scattering versus the nanoparticle radius presented in Fig. 4(a) confirms that the Rabi splitting due to the dark mode coupling is preserved for a wide range of the nanoparticle size. In this plot, the QE detuning was placed in resonance with the dark pseudomode so that $\delta_{E}=0.35 \mathrm{eV}$, for the smallest radius of $5 \mathrm{~nm}$. The observed effect appears to be much more sensitive to the surface-emitter separation $h$, as Fig. 4(b) indicates. The Rabi splitting in the vicinity of the bright mode is sustained only up to $2 \mathrm{~nm}$ separation and disappears for larger distances, where only the uncoupled bright mode and the emitter contribute to scattering. This behavior originates from the dark pseudomode strong dependence on $h$. With increasing $h$, the coupling to the dark mode quickly diminishes, leaving only the signatures of the bright mode in the spectrum.

In addition, we demonstrate the photon blockade for $R=$ $5 \mathrm{~nm}$ versus $h$. The results are shown in Fig. 4(c), where we plot the scattered photon statistics for zero delay, i.e., the second-order correlation function (12). We show that anti- bunched light $\left(g_{\omega}^{(2)}(0)<1\right)$ is produced following the LP since it is a mixture of the dark plexciton and the bright mode, while slightly bunched light $\left(g_{\omega}^{(2)}(0)>1\right)$ appears on the dark plexciton UP. We underline here that the antibunching is resulting from strong interactions with both dark and bright modes, even if the dark pseudomode is usually thought of as being detrimental for the radiative properties of the system. Also, even if the QE is being hybridized with two plasmon modes, the photon statistics shows clear antibunching, indicating the robustness of single-photon emission in this scheme. Finally, despite the resonances being in the near UV, the single-photon emission line is shown to be red shifted so that it can be seen in the visible. We further discuss this effect by varying manually the Hamiltonian with similar parameters. Results are shown in Figs. 5 and 6. Figure 5(a) shows the scattering when the QE frequency is tuned in resonance with the dark pseudomode $\omega_{E}=\omega_{D}$, and one can see an anticrossing between the LP and MP around $g_{D}=0.5 \mathrm{eV}$. The UP is here not very visible since it is strongly detuned with the bright mode. When the MP and LP are strongly coupled, an antibunching line appears in Fig. 5(b), and the latter is further red shifted when $g_{D}$ and $g_{B}$ increase. In Fig. 6, we plot the same data with the same parameters except for the QE frequency that is artificially swept in order to match the optimal frequency $\omega_{E}^{\mathrm{opt}}=\omega_{B}+\delta_{E}^{\mathrm{opt}}$, hence maintaining the optimal Rabi splitting between the LP and the MP. One can observe that for very high dark coupling
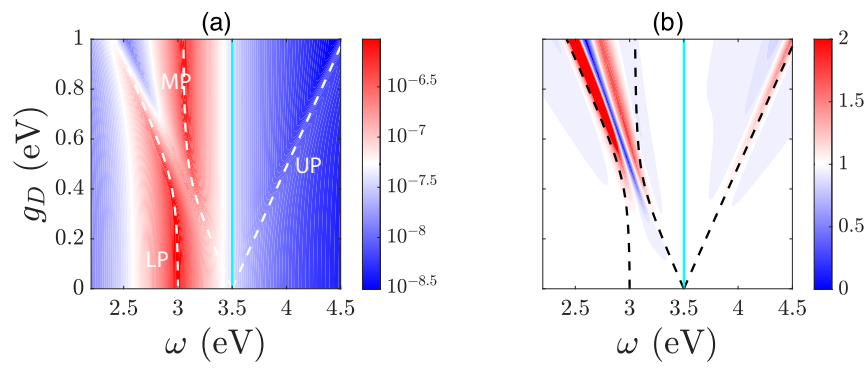

FIG. 5. (a) Log-scale scattering intensity of the coupled QE bright- and dark-mode system vs weak drive frequency $\omega$. Here $\omega_{E}=$ $\omega_{D}=3.5 \mathrm{eV}$ (light blue line), $\omega_{B}=3 \mathrm{eV}$ and $g_{D}, g_{B}$ are linearly swept from 0 to $1 \mathrm{eV}$ and 0 to $0.3 \mathrm{eV}$, respectively. (b) Zero-delay second-order coherence function $g_{\omega}^{(2)}(0)$ with the same parametrization as in panel (a). Lower, middle, and upper polariton (LP, MP, UP) lines are shown in dashed solid lines. Decay rates were fixed $\gamma_{B}=\gamma_{D}=0.2 \mathrm{eV}$ and $\gamma_{E}=0.1 \mathrm{eV}$. 

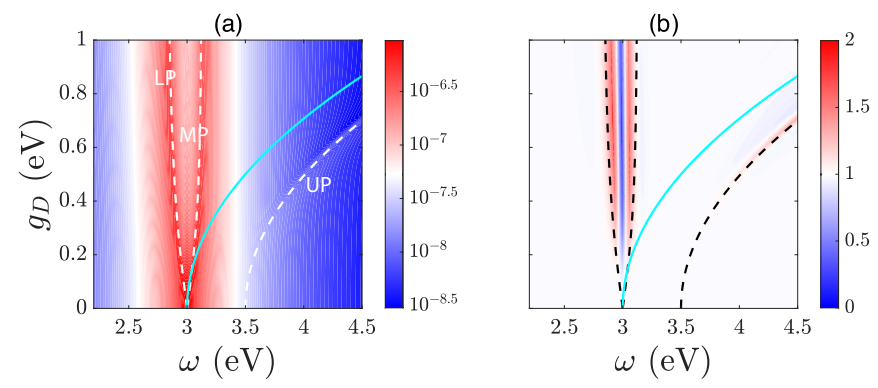

FIG. 6. (a) Log-scale scattering intensity of the coupled QE bright- and dark-mode system vs weak drive frequency $\omega$. All parameters are the same as in Fig. 5 except for $\omega_{E}$, which is here taken to be the optimal frequency $\omega_{E}^{\text {opt }}=\omega_{B}+\delta_{E}^{\text {opt }}$. (b) Zero-delay second-order coherence function $g_{\omega}^{(2)}(0)$ with the same parametrization as in (a). Lower, middle, and upper polariton (LP, MP, UP) lines are shown in dashed solid lines.

strengths $g_{D}$ the UP and the emitter are very far detuned to the blue but the antibunching line in Fig. 6(b) remains unchanged.

\section{CONCLUSION}

We have presented a scheme for realizing strong lightmatter coupling with use of a high-energy electronic transition of a large oscillator strength quantum emitter. Exploiting the nonradiative modes of a plasmonic cavity, the high-energy transition can be tuned to lower energies, where it can couple with the bright plasmon cavity mode leading to observable vacuum Rabi splitting in the scattering spectrum. Results were predicted by a simple model and verified with the use of an effective master equation approach for realistic coupling parameters and cavity geometries. Quantum nonlinearities were also shown with the use of the second-order coherence function and found to be robust with respect to dark mode coupling. UV transitions of colloidal quantum dots or $\mathrm{C}$ excitons of transition-metal dichalcogenides are possible candidates for the proposed approach toward strong coupling [24,58]. This work could help in the design of QE-plasmon coupling schemes toward the realization of efficient room-temperature strong coupling and quantum nonlinearities.

\section{ACKNOWLEDGMENT}

The authors acknowledge support from the Swedish Research Council (VR Grant No. 2016-06059).

\section{APPENDIX A: TEMPORAL COUPLED MODE THEORY}

In the framework of coupled mode theory, the system response is described by a ket-vector with complex amplitudes $|a\rangle=\left(c_{B}, c_{D}, c_{E}\right)^{T}$, where the subscripts $B, D, E$ denote corresponding amplitudes for the bright mode, the dark pseudomode, and the QE, respectively. The dynamics of the amplitudes is governed by the Schrödinger-like equation

$$
i \frac{d|a\rangle}{d t}=\widehat{H}|a\rangle+s_{+}|\kappa\rangle, \quad|\kappa\rangle=\left(\kappa_{B}, \kappa_{D}, \kappa_{E}\right)^{T},
$$

where $\widehat{H}$ is the system Hamiltonian, $|\kappa\rangle$ is the mode-radiation coupling constants vector with components $\kappa_{j}=\sqrt{\gamma_{j}^{\mathrm{rad}}}, \gamma_{j}^{\mathrm{rad}}$ are the radiative decay rates of each mode, and $s_{+}$is the incident wave amplitude. The Hamiltonian of the three-mode system reads

$$
\widehat{H}=\left(\begin{array}{ccc}
\omega_{B}-i \gamma_{B} / 2 & 0 & g_{B}-i \gamma_{\text {ind }} \\
0 & \omega_{D}-i \gamma_{D} / 2 & g_{D} \\
g_{B}-i \gamma_{\text {ind }} & g_{D} & \omega_{E}-i \gamma_{E} / 2
\end{array}\right),
$$

where $\omega_{j}, \gamma_{j}$ stand for the eigenfrequencies and total decay rates of each mode, respectively. The non-Hermitian term with $\gamma_{\text {ind }}=\sqrt{\gamma_{B}^{\mathrm{rad}} \gamma_{E}^{\mathrm{rad}} / 4}$ comes from the far-field (indirect) coupling of the bright mode with the QE [59] and can be neglected when the QE radiative decay is much smaller than that of the bright mode. For a harmonic excitation at frequency $\omega$, the steady-state solution of Eq. (1) reads $|a\rangle=\frac{|\kappa\rangle s_{+}}{i(\widehat{H}-\omega)}$. Finally, the amplitude of the scattered signal in the steadystate regime is given by $s_{-}=\langle\kappa \mid a\rangle$.

\section{APPENDIX B: BRIGHT- AND DARK-MODE DECOMPOSITION: EFFECTIVE HAMILTONIAN}

In the rotating-wave approximation, the non-Hermitian Hamiltonian for the nanosphere-emitter system reads, using the spherical orthogonal mode decomposition,

$$
\begin{aligned}
\widetilde{H}_{\text {m.d. }}= & \left(\omega_{E}-i \frac{\gamma_{E}}{2}\right) \widehat{\sigma}_{+} \widehat{\sigma}_{-}+\sum_{n=1}^{\infty}\left(\omega_{n}-i \frac{\gamma_{n}}{2}\right) \widehat{a}_{n}^{\dagger} \widehat{a}_{n} \\
& +\sum_{n=1}^{\infty} g_{n}\left(\widehat{a}_{n}^{\dagger} \widehat{\sigma}_{-}+\widehat{a}_{n} \widehat{\sigma}_{+}\right)
\end{aligned}
$$

where $\omega_{E}$ is the transition frequency of the QE, $\widehat{\sigma}_{-}, \widehat{\sigma}_{+}$is its lowering and raising operators, respectively, and $\gamma_{E}$ is its total decay rate. The plasmonic field is modeled with creation and annihilation operators $\widehat{a}_{n}^{\dagger}, \widehat{a}_{n}$ associated with frequencies $\omega_{n}$ and decay rates $\gamma_{n}$. Each $n$ mode corresponds to a specific plasmon resonance: $n=1$ is the dipolar mode, $n=2$ is the quadrupolar, $n=3$ is the octupolar, and so on, and each mode $n$ couples to the QE with coupling rate $g_{n}$. In the case of a spherical nanoparticle in the local-response approximation $[41,53,54]$, the dipole mode is usually well separated from the higher order modes $n \geqslant 2$ and the latter, being quasidegenerate, behave effectively as a large pseudomode when the emitter is very close to the surface of the sphere. The quasidegeneracy of the higher order modes is valid in the local-response approximation, where nonlocal effects are disregarded. This may not be true for very small spheres, whose spontaneous emission spectrum in the vicinity of the metal surface then present nondegenerate higher order modes [53]. However, for simplicity we will use the local-response approximation for silver spheres of radii between 5 and 20 $\mathrm{nm}$. In the case of degenerate modes, however, one could still apply our formalism using the quadrupole mode as the dark mode that will couple to the QE. In the following, we note $\omega_{1} \equiv \omega_{B}, \gamma_{1} \equiv \gamma_{B}, \widehat{a}_{1} \equiv \widehat{a}_{B}, g_{1} \equiv g_{B}$, and

$$
\widehat{a}_{D}=\frac{1}{g_{D}} \sum_{n \geqslant 2}^{\infty} g_{n} \widehat{a}_{n} .
$$


The commutation relation of the original modes $\left[\widehat{a}_{n}, \widehat{a}_{m}^{\dagger}\right]=$ $\delta_{n m}$ leads to the effective dark coupling to be $g_{D}=$ $\left(\sum_{n \geqslant 2}^{\infty} g_{n}^{2}\right)^{1 / 2}$ in order to have the dark modes normalized and the right commutation relation $\left[\widehat{a}_{D}, \widehat{a}_{D}^{\dagger}\right]=1$. The effective non-Hermitian system Hamiltonian then has the form

$$
\begin{aligned}
\widetilde{H}_{S}= & \left(\omega_{E}-i \frac{\gamma_{E}}{2}\right) \widehat{\sigma}_{+} \widehat{\sigma}_{-}+\left(\omega_{B}-i \frac{\gamma_{B}}{2}\right) \widehat{a}_{B}^{\dagger} \widehat{a}_{B} \\
& +\left(\omega_{D}-i \frac{\gamma_{D}}{2}\right) \widehat{a}_{D}^{\dagger} \widehat{a}_{D}+g_{B}\left(\widehat{a}_{B}^{\dagger} \widehat{\sigma}_{-}+\widehat{a}_{B} \widehat{\sigma}_{+}\right) \\
& +g_{D}\left(\widehat{a}_{D}^{\dagger} \widehat{\sigma}_{-}+\widehat{a}_{D} \widehat{\sigma}_{+}\right) .
\end{aligned}
$$

The resonance $\omega_{D}$ and the decay rate $\gamma_{D}$ are obtained by fitting the pseudomode by a Lorentzian function and extracting its maximum position and full width at half maximum. The calculation of the Lorentzian-fitted local density of states (LDOS) from the Green's tensor approach then yields the parameters $\left(g_{B}, g_{D}, \omega_{B}, \omega_{D}, \gamma_{B}, \gamma_{D}\right)$ that appear in the nonHermitian Hamiltonian $[32,57,60]$. The spontaneous emission enhancement factor seen in Fig. 1(c) is then computed through the following formula:

$$
F_{P}(\omega)=\sum_{n} \frac{\mathbf{u} \cdot \operatorname{Im}\left\{\overline{\overline{\mathbf{G}}}_{n}\left(\mathbf{r}_{E}, \mathbf{r}_{E}, \omega\right)\right\} \mathbf{u}}{\mathbf{u} \cdot \operatorname{Im}\left\{\overline{\overline{\mathbf{G}}}_{\text {free }}\left(\mathbf{r}_{E}, \mathbf{r}_{E}, \omega\right)\right\} \mathbf{u}},
$$

where $\mathbf{u}$ is the unit vector oriented along the $\mathrm{QE}$ dipole moment, $\mathbf{r}_{E}$ is the position of the $\mathrm{QE}, \overline{\overline{\mathbf{G}}}_{n}\left(\mathbf{r}, \mathbf{r}^{\prime}, \omega\right)$ is the $n$th mode Green's tensor, and $\overline{\overline{\mathbf{G}}}_{\text {free }}\left(\mathbf{r}, \mathbf{r}^{\prime}, \omega\right)$ is the Green's tensor corresponding to a $\mathrm{QE}$ in free space.

\section{APPENDIX C: $3 \times 3$ HAMILTONIAN DESCRIPTION: PARTIAL DIAGONALIZATION AND BRIGHT-MODE SPLITTING}

The Hermitian part of the Hamiltonian (B1) can be written in a matrix form considering the single excitation basis: One excitation only is exchanged between the QE transition and the plasmon modes. Let the matrix form of the Hamiltonian generally be written in the basis $\left\{|e, 0,0\rangle,\left|g, 1_{D}, 0\right\rangle,\left|g, 0,1_{B}\right\rangle\right\}$ :

$$
\mathrm{H}=\left[\begin{array}{ccc}
0 & g_{D} & g_{B} \\
g_{D} & \Delta_{D} & 0 \\
g_{B} & 0 & \Delta_{B}
\end{array}\right],
$$

where we wrote the Hamiltonian in a rotating frame with respect to $\omega_{E}$, so that $\Delta_{D, B}=\omega_{D, B}-\omega_{E}$. When an excitonic transition strongly couples to a plasmon mode, two polaritons [lower polariton (LP) and upper polariton (UP)] are formed and it is convenient to diagonalize the Hamiltonian block involving them. Also, writing the Hamiltonian in the basis of the polaritons enables to understand how the latter effectively couple to the other components of the Hamiltonian.

\section{Diagonalization of the strongly coupled block}

In the following, we consider the block $\Pi$ of the Hamilto$\operatorname{nian}(\mathrm{C} 1)$ :

$$
\Pi=\left[\begin{array}{cc}
0 & g_{D} \\
g_{D} & \Delta_{D}
\end{array}\right]
$$

The eigenvalues of this block are the following:

$$
\begin{aligned}
& \delta_{ \pm}=\frac{1}{2}\left(\Delta_{D} \pm \Upsilon\right), \\
& \Upsilon=\sqrt{\Delta_{D}^{2}+4 g_{D}^{2}} .
\end{aligned}
$$

It is convenient to introduce the angle $\theta$ parametrized as follows:

$$
\begin{aligned}
& \cos \theta=\frac{\Delta_{D}}{\Upsilon}, \\
& \sin \theta=\frac{2 g_{D}}{\Upsilon}, \\
& \tan \theta=\frac{2 g_{D}}{\Delta_{D}} .
\end{aligned}
$$

It is then possible to write the block with respect to $\theta$ :

$$
\begin{aligned}
\Pi & =\Upsilon\left[\begin{array}{cc}
0 & \frac{1}{2} \sin \theta \\
\frac{1}{2} \sin \theta & \cos \theta
\end{array}\right] \\
& =\Upsilon\left[\begin{array}{cc}
0 & \sin \frac{\theta}{2} \cos \frac{\theta}{2} \\
\sin \frac{\theta}{2} \cos \frac{\theta}{2} & \cos ^{2} \frac{\theta}{2}-\sin ^{2} \frac{\theta}{2}
\end{array}\right] .
\end{aligned}
$$

The eigenvalues can also be expressed in terms of the parametrized angle,

$$
\delta_{ \pm}=\Upsilon\left\{\begin{array}{c}
\cos ^{2} \frac{\theta}{2} \\
-\sin ^{2} \frac{\theta}{2}
\end{array}\right\}
$$

which enables to write the transformation diagonalizing the block as

$$
\mathrm{T}^{\dagger} \Pi \mathrm{T}=\Upsilon\left[\begin{array}{cc}
-\sin ^{2} \frac{\theta}{2} & 0 \\
0 & \cos ^{2} \frac{\theta}{2}
\end{array}\right] .
$$

Using the decomposition of the block $\Pi$ in terms of $\theta$, the unitary transformation $\mathrm{T}$ containing the eigenvectors $\left|\phi_{ \pm}\right\rangle$ associated with the eigenvalues $\delta_{ \pm}$reads

$$
\mathrm{T}=\left[\left|\phi_{-}\right\rangle,\left|\phi_{+}\right\rangle\right]=\left[\begin{array}{cc}
\cos \frac{\theta}{2} & \sin \frac{\theta}{2} \\
-\sin \frac{\theta}{2} & \cos \frac{\theta}{2}
\end{array}\right] .
$$

Once it is diagonalized, the $\Pi$ block is expressed in the basis of the polaritons $\left\{\left|\phi_{-}\right\rangle,\left|\phi_{+}\right\rangle\right\}$. The LP is associated with the subscript $(-)$ while the UP is associated with the subscript (+).

\section{Partial diagonalization of the $3 \times 3$ Hamiltonian}

In this section, we diagonalize partially the Hamiltonian (C1) using the results of the previous section. To do so, we create the following transformation:

$$
\mathrm{T}_{3}=\left[\begin{array}{cc|c}
\cos \frac{\theta}{2} & \sin \frac{\theta}{2} & 0 \\
-\sin \frac{\theta}{2} & \cos \frac{\theta}{2} & 0 \\
\hline 0 & 0 & 1
\end{array}\right],
$$

which transforms only the $\Pi$ block of the Hamiltonian. Changing the frame of reference of the Hamiltonian using this 


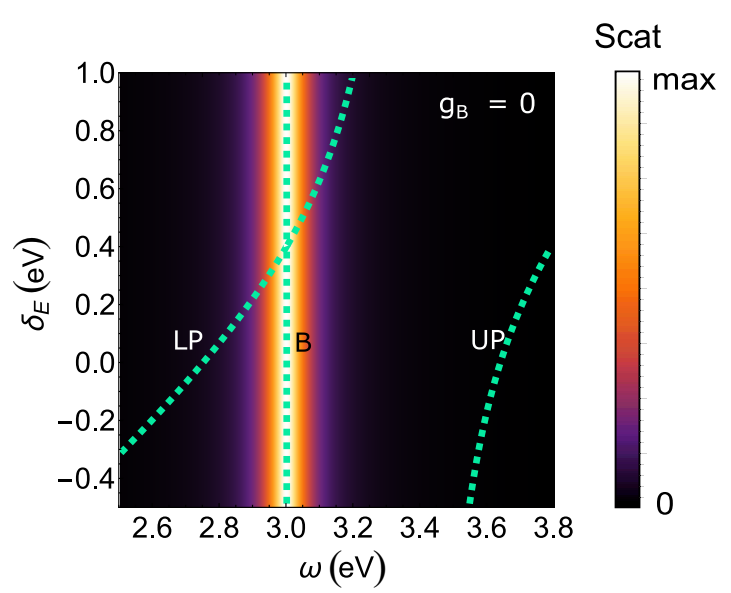

FIG. 7. Map of simulated scattering spectra vs emitter detuning $\delta_{E}$ for the exemplary $3 \times 3$ system. The parameters are the same as in Fig. 3(b), except that $g_{B}=0$.

transformation, we get

$$
\mathrm{T}_{3}^{\dagger} \mathrm{HT}_{3}=\left[\begin{array}{cc|c}
\delta_{-} & 0 & g_{B} \cos \frac{\theta}{2} \\
0 & \delta_{+} & g_{B} \sin \frac{\theta}{2} \\
\hline g_{B} \cos \frac{\theta}{2} & g_{B} \sin \frac{\theta}{2} & \Delta_{B}
\end{array}\right] .
$$

This Hamiltonian describes the interaction of both polaritons with a third state. Originally, only one of the polariton components is coupled to this state with coupling strength $g_{B}$, but the polaritons both couple to it with $g_{B} \cos \frac{\theta}{2}$ for $(-)$ and $g_{B} \sin \frac{\theta}{2}$ for $(+)$. Another consideration is how resonant the final system is. If the separation $\delta_{+}-\delta_{-}=\Upsilon$ is larger than the linewidth of the third state, then only one polariton will couple efficiently with it. Finally, let us have a closer look at the sine and cosine factors. Using both (C3a) and (C6), we find that these factors have the form

$$
\begin{aligned}
& \sin \frac{\theta}{2}=\frac{1}{\sqrt{2}} \sqrt{1-\frac{\Delta_{D}}{\sqrt{\Delta_{D}^{2}+4 g_{D}^{2}}}}, \\
& \cos \frac{\theta}{2}=\frac{1}{\sqrt{2}} \sqrt{1+\frac{\Delta_{D}}{\sqrt{\Delta_{D}^{2}+4 g_{D}^{2}}}} .
\end{aligned}
$$

\section{Optimal QE frequency and bright-mode splitting}

In our system, the dark mode is located in the blue part of the spectrum. If the splitting between the dark mode and

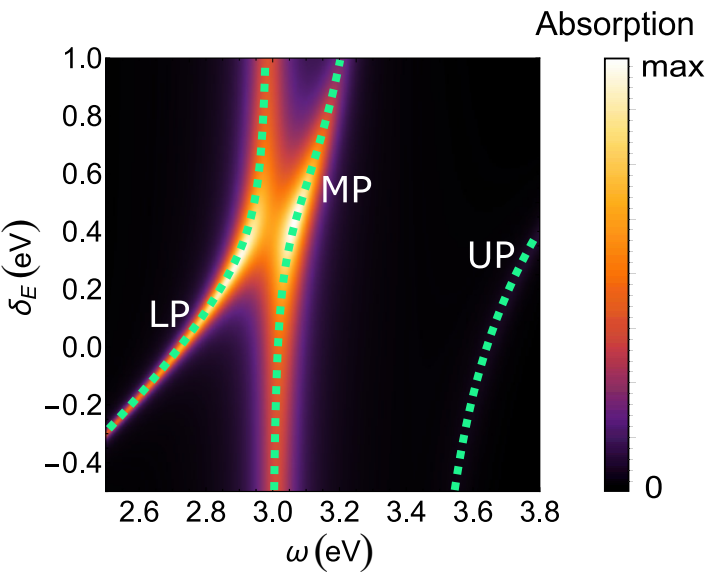

FIG. 8. Map of simulated absorption spectra vs emitter detuning $\delta_{E}$ for the exemplary $3 \times 3$ system. The parameters are the same as in Fig. 3(b).

the $\mathrm{QE}$ is large enough, we expect the lower polariton to approach the resonance frequency of the bright mode and start interacting with it. If we look at the Hamiltonian in the partially diagonalized basis $(\mathrm{C} 10)$, we see that the resonance happens for $\delta_{-}=\Delta_{B}$. We call the optimal QE-bright-mode detuning $\Delta_{B}^{\text {opt }}$ and using equations (C3a) we find its value:

$$
\Delta_{B}^{\mathrm{opt}}=-\frac{g_{D}^{2}}{\Delta_{D}-\Delta_{B}}=-\frac{g_{D}^{2}}{\omega_{D}-\omega_{B}} .
$$

The vacuum Rabi splitting of the bright mode is then calculated from Eqs. (C10) and (C11) and we get

$$
\Omega_{\text {bright }}=2 g_{B} \cos \frac{\theta}{2}=\sqrt{2} g_{B} \sqrt{1+\frac{\Delta_{D}}{\sqrt{\Delta_{D}^{2}+4 g_{D}^{2}}}} .
$$

This Rabi splitting corresponds to the one observed in Figs. 3 and 4. In addition to Fig. 3, we plot the scattering map for $g_{B}=0$ (see Fig. 7): No splitting is observed for mode B but we can see the dark-mode QE vacuum Rabi splitting and its corresponding LP and UP. Finally, we show the absorption spectra versus B-mode QE detuning in Fig. 8. The latter is computed using the steady-state and

$$
\begin{gathered}
\text { Abs }=\left\langle\psi_{\text {s.s. }}\left|\widehat{b}^{\dagger} \widehat{b}\right| \psi_{\text {s.s. }}\right\rangle \\
\widehat{b}=\sqrt{\gamma_{B}^{\text {nonrad }}} \widehat{a}_{B}+\sqrt{\gamma_{D}^{\text {nonrad }}} \widehat{a}_{D} .
\end{gathered}
$$

where we here set $\gamma_{B}^{\text {nonrad }}=0.01 \mathrm{eV}, \gamma_{D}^{\text {nonrad }}=0.1 \mathrm{eV}$, and we here neglected the non-radiative decay of the $\mathrm{QE}$.
[1] M. O. Scully and M. S. Zubairy, Quantum Optics (Cambridge University Press, Cambridge, UK, 1997), p. 656.

[2] M. S. Tame, K. R. McEnery, S. K. Ozdemir, J. Lee, S. A. Maier, and M. S. Kim, Nat. Phys. 9, 329 (2013).

[3] N. T. Fofang, N. K. Grady, Z. Fan, A. O. Govorov, and N. J. Halas, Nano Lett. 11, 1556 (2011).

[4] G. Khitrova, H. M. Gibbs, M. Kira, S. W. Koch, and A. Scherer, Nat. Phys. 2, 81 (2006).
[5] J. M. Fink, M. Goppl, M. Baur, R. Bianchetti, P. J. Leek, A. Blais, and A. Wallraff, Nature (London) 454, 315 (2008).

[6] P. Törmä and W. L. Barnes, Rep. Prog. Phys. 78, 013901 (2015).

[7] D. G. Baranov, M. Wersall, J. Cuadra, T. J. Antosiewicz, and T. Shegai, ACS Photon. 5, 24 (2018).

[8] J. Schachenmayer, C. Genes, E. Tignone, and G. Pupillo, Phys. Rev. Lett. 114, 196403 (2015). 
[9] J. A. Hutchison, T. Schwartz, C. Genet, E. Devaux, and T. W. Ebbesen, Angew. Chem., Int. Ed. 51, 1592 (2012).

[10] A. Thomas, J. George, A. Shalabney, M. Dryzhakov, S. J. Varma, J. Moran, T. Chervy, X. Zhong, E. Devaux, C. Genet, J. A. Hutchison, and T. W. Ebbesen, Angew. Chem. Int. Ed. 55, 11462 (2016).

[11] F. Herrera and F. C. Spano, Phys. Rev. Lett. 116, 238301 (2016).

[12] J. Galego, F. J. Garcia-Vidal, and J. Feist, Nat. Commun. 7, 13841 (2016).

[13] B. Munkhbat, M. Wersäll, D. G. Baranov, T. J. Antosiewicz, and T. Shegai, Sci. Adv. 4, eaas9552 (2018).

[14] K. M. Birnbaum, A. Boca, R. Miller, A. D. Boozer, T. E. Northup, and H. J. Kimble, Nature (London) 436, 87 (2005).

[15] D. Englund, A. Faraon, I. Fushman, N. Stoltz, P. Petroff, and J. Vuckovic, Nature (London) 450, 857 (2007).

[16] T. Yoshie, A. Scherer, J. Hendrickson, G. Khitrova, H. M. Gibbs, G. Rupper, C. Ell, O. B. Shchekin, and D. G. Deppe, Nature (London) 432, 200 (2004).

[17] J. P. Reithmaier, G. Sek, A. Löffler, C. Hofmann, S. Kuhn, S. Reitzenstein, L. V. Keldysh, V. D. Kulakovskii, T. L. Reinecke, and A. Forchel, Nature (London) 432, 197 (2004).

[18] R. Chikkaraddy, B. de Nijs, F. Benz, S. J. Barrow, O. A. Scherman, E. Rosta, A. Demetriadou, P. Fox, O. Hess, and J. J. Baumberg, Nature (London) 535, 127 (2016).

[19] K. Santhosh, O. Bitton, L. Chuntonov, and G. Haran, Nat. Commun. 7, 11823 (2016).

[20] H. Groß, J. M. Hamm, T. Tufarelli, O. Hess, and B. Hecht, Sci. Adv. 4, eaar4906 (2018).

[21] H. Leng, B. Szychowski, M.-C. Daniel, and M. Pelton, Nat. Commun. 9, 4012 (2018).

[22] M. Pelton, S. D. Storm, and H. Leng, Nanoscale 11, 14540 (2019).

[23] B. Rousseaux, D. G. Baranov, M. Käll, T. Shegai, and G. Johansson, Phys. Rev. B 98, 045435 (2018).

[24] C. A. Leatherdale, W.-K. Woo, F. V. Mikulec, and M. G. Bawendi, J. Phys. Chem. B 106, 7619 (2002).

[25] W. W. Yu, L. Qu, W. Guo, and X. Peng, Chem. Mat. 15, 2854 (2003).

[26] G. Wang, A. Chernikov, M. M. Glazov, T. F. Heinz, X. Marie, T. Amand, and B. Urbaszek, Rev. Mod. Phys. 90, 021001 (2018).

[27] M. W. Knight, N. S. King, L. Liu, H. O. Everitt, P. Nordlander, and N. J. Halas, ACS Nano 8, 834 (2013).

[28] E. Eizner, O. Avayu, R. Ditcovski, and T. Ellenbogen, Nano Lett. 15, 6215 (2015).

[29] T. P. Rossi, T. Shegai, P. Erhart, and T. J. Antosiewicz, Nat. Commun. 10, 3336 (2019).

[30] M. Liu, T.-W. Lee, S. K. Gray, P. Guyot-Sionnest, and M. Pelton, Phys. Rev. Lett. 102, 107401 (2009).

[31] A. Delga, J. Feist, J. Bravo-Abad, and F. J. Garcia-Vidal, Phys. Rev. Lett. 112, 253601 (2014).

[32] B. Rousseaux, D. Dzsotjan, G. Colas des Francs, H. R. Jauslin, C. Couteau, and S. Guérin, Phys. Rev. B 93, 045422 (2016).

[33] H. Varguet, B. Rousseaux, D. Dzsotjan, H.-R. Jauslin, S. Guérin, and G. C. des Francs, Opt. Lett. 41, 4480 (2016).

[34] R.-Q. Li, F. J. García-Vidal, and A. I. Fernández-Domínguez, ACS Photon. 5, 177 (2018).
[35] A. Castellini, H. R. Jauslin, B. Rousseaux, D. Dzsotjan, G. Colas des Francs, A. Messina, and S. Guérin, Eur. Phys. J. D 72, 223 (2018).

[36] H. Varguet, B. Rousseaux, D. Dzsotjan, H. R. Jauslin, S. Guérin, and G. C. des Francs, J. Phys. B: At., Mol. Opt. Phys. 52, 055404 (2019).

[37] A. Cuartero-González and A. I. Fernández-Domínguez, Phys. Rev. B 101, 035403 (2020).

[38] A. L. Koh, K. Bao, I. Khan, W. E. Smith, G. Kothleitner, P. Nordlander, S. A. Maier, and D. W. McComb, ACS Nano 3, 3015 (2009).

[39] S. J. Barrow, D. Rossouw, A. M. Funston, G. A. Botton, and P. Mulvaney, Nano Lett. 14, 3799 (2014).

[40] A. B. Yankovich, B. Munkhbat, D. G. Baranov, J. Cuadra, E. Olsén, H. Lourenço-Martins, L. H. G. Tizei, M. Kociak, E. Olsson, and T. Shegai, Nano Lett. 19, 8171 (2019).

[41] G. P. Zouros, G. D. Kolezas, N. A. Mortensen, and C. Tserkezis, Phys. Rev. B 101, 085416 (2020).

[42] O. Bitton, S. N. Gupta, L. Houben, M. Kvapil, V. Křápek, T. Šikola, and G. Haran, Nat. Commun. 11, 487 (2020).

[43] K. Kolwas, S. Demianiuk, and M. Kolwas, J. Chem. Phys. 106, 8436 (1997).

[44] K. Du, P. Li, K. Gao, H. Wang, Z. Yang, W. Zhang, F. Xiao, S. J. Chua, and T. Mei, J. Phys. Chem. Lett. 10, 4699 (2019).

[45] D. E. Gómez, Z. Q. Teo, M. Altissimo, T. J. Davis, S. Earl, and A. Roberts, Nano Lett. 13, 3722 (2013).

[46] L. Novotny and B. Hecht, Principles of Nano-optics (Cambridge University Press, Cambridge, UK, 2012).

[47] P. Anger, P. Bharadwaj, and L. Novotny, Phys. Rev. Lett. 96, 113002 (2006).

[48] A. Cuartero-González and A. I. Fernández-Domínguez, ACS Photon. 5, 3415 (2018).

[49] H. Haus, Waves and Fields in Optoelectronics (Prentice Hall, Englewood Cliffs, NJ, 1984).

[50] S. Fan, W. Suh, and J. D. Joannopoulos, J. Opt. Soc. Am. B 20 , 569 (2003).

[51] R. Sáez-Blázquez, J. Feist, A. I. Fernández-Domínguez, and F. J. García-Vidal, Optica 4, 1363 (2017).

[52] M. I. Stockman, Opt. Expr. 19, 22029 (2011).

[53] T. Christensen, W. Yan, S. Raza, A.-P. Jauho, N. Asger Mortensen, and M. Wubs, ACS Nano 8, 1745 (2014).

[54] C. Tserkezis, M. Wubs, and N. Asger Mortensen, ACS Photon. 5, 133 (2018).

[55] E. D. Palik, Handbook of Optical Constants of Solids (Academic Press, New York, 1998), Vol. 3.

[56] M. D. Leistikow, J. Johansen, A. J. Kettelarij, P. Lodahl, and W. L. Vos, Phys. Rev. B 79, 045301 (2009).

[57] D. Dzsotjan, B. Rousseaux, H. R. Jauslin, G. C. des Francs, C. Couteau, and S. Guérin, Phys. Rev. A 94, 023818 (2016).

[58] Y. Li, A. Chernikov, X. Zhang, A. Rigosi, H. M. Hill, A. M. van der Zande, D. A. Chenet, E.-M. Shih, J. Hone, and T. F. Heinz, Phys. Rev. B 90, 205422 (2014).

[59] W. Suh, Z. Wang, and S. Fan, IEEE J. Quant. Electron. 40, 1511 (2004).

[60] J. Hakami, L. Wang, and M. S. Zubairy, Phys. Rev. A 89, 053835 (2014). 